\title{
Visual object individuation occurs over object wholes, parts, and even holes
}

\author{
Katharine B. Porter ${ }^{1}$ - Veronica Mazza ${ }^{2,3}$ - Annie Garofalo ${ }^{1}$ - Alfonso Caramazza ${ }^{1,2}$
}

Published online: 16 February 2016

(C) The Psychonomic Society, Inc. 2016

\begin{abstract}
Segmentation of the world into meaningful units has typically been described as object individuation, emphasizing the spatially disconnected quality that comes as a result of objecthood. This segmentation can occur rapidly, even in parallel for multiple objects. It remains unclear whether objecthood is a necessary requirement for parallel individuation, or whether target features in distinct locations, such as object parts, may also be individuated in parallel. In a series of six experiments, we used a rapid enumeration task to test whether subitizing, a phenomenon believed to result from parallel individuation, occurs over object parts. We found that subitizing and individuation occur over connected object parts as well as physically separate objects of varied shapes and sizes. We also observed subitizing when target items are indents, features intrinsic to the shape of the object, and when cues for occlusion were removed. The results of these studies suggest that parallel individuation is not bound to objecthood, and can occur over object parts existing in separate locations.
\end{abstract}

Keywords Object individuation · Subitizing · Enumeration · Object features $\cdot$ Visual segmentation $\cdot$ Multiple object individuation

Katharine B. Porter

kporter@fas.harvard.edu

1 Department of Psychology, Harvard University, William James Hall Room 936, 33 Kirkland St, Cambridge, MA 02138, USA

2 Center for Mind/Brain Sciences (CIMeC), University of Trento, 38123 Trento, Italy

3 IRCCS Centro San Giovanni di Dio Fatebenefratelli, 25125 Brescia, Italy
The human mind is remarkable; it has the ability to track objects through time and space (Scholl, Pylyshyn, \& Feldman, 2001), enumerate sets of items (Trick \& Pylyshyn, 1994), calculate the frequency of events (Brase, Cosmides, \& Tooby, 1998), and recognize objects while maintaining their identity under variable perceptual conditions (Kraft \& Brainard, 1999; Marr, 1982). While the objectives of each of these skills are quite varied, they all share a commonality: they depend on a mechanism that separates figure from ground and parses the visual field into separate entities. The question remains - what are those entities? In most circumstances, this visual segmentation and selection is referred to as individuation, and the nebulous term "object" fills in the blank for "of what?"

Parallel individuation is often measured by performance on an enumeration task. This takes the form of accuracy and speed when measured in adults. The phenomenon "subitizing" is observed in these two measures, where subjects rapidly and accurately enumerate small sets of physically separate objects without much cost for each additional item, seemingly individuating the items in parallel (Kaufman, Lord, Reese, \& Volkmann, 1949; Trick \& Pylyshyn, 1994). This manifests as a substantially smaller increase in reaction time (RT) and error rate between two consecutive numbers within the "subitizing range" ( 1 to $\sim 4)$ than the increase in RT and errors between consecutive numbers in the "counting range" (about $5+$ ). This creates an "elbow" in response functions where a sharp change in slope occurs at $\sim 4$ items, depending on the individual's subitizing range (Akin \& Chase, 1978).

Several theories exist as to why there seems to be a processing advantage for small sets of numbers. One view is that the rapid enumeration of small sets is merely a result of highly accurate pattern matching (Mandler \& Shebo, 1982; Palomares \& Egeth, 2010); this theory has since been 
expanded to include the element of similarity, with the faster and more accurate responses for lower numbers reflecting the higher within-number similarity and between-number dissimilarity of the different display configurations for set sizes within the subitizing range as compared to large set sizes (Logan \& Zbrodoff, 2003).

While the studies described above tend to characterize subitization as a processing advantage due to discriminability or pattern matching, several other theories suggest that a separate mechanism exists for selecting and processing small sets of items. One of the most pervasive is the "FINST" theory, named after the "Fingers of INSTantiation," or indices, that point out or individuate items for further processing (Pylyshyn, 1989). This theory suggests that distinctive items in the visual field are selected preattentively and assigned to a limited number $(\sim 4)$ of indices. These indices have no knowledge of the item's identity or features, but mark the location for further processing by attention. Once an index has been assigned, it will maintain its assignment even throughout the motion (Pylyshyn \& Storm, 1988) or occlusion (Scholl \& Pylyshyn, 1999) of its target. While the FINST theory originated from studies of multiple object tracking (MOT), subitizing is believed to draw upon the same individuation resource as MOT. Simultaneous MOT and enumeration reflects a shared limit across the two tasks of about four items (Chesney \& Haladjian, 2011). Furthermore, both subitizing (Railo, 2014) and MOT (Alvarez \& Cavanagh, 2005) demonstrate a bilateral presentation advantage. Both of these findings support the view of a shared parallel individuation mechanism across the two tasks.

To better understand the mechanism underlying parallel individuation, it is important to consider under what conditions individuation can occur. Humans are capable of object individuation early in development (Spelke, Kestenbaum, Simons, \& Wein, 1995), and in this context, the definition of "object" is given as "...unitary, bounded, and persisting objects...operating in accord with the principles of cohesion, boundedness, rigidity, and no action at a distance" (Spelke, 1990). Spatiotemporal information (Spelke et al., 1995) and connectivity seem to be the primary cues infants use to individuate objects through 10 months of age (Xu, Carey, \& Welch, 1999), and not until they are 12 months old do they start using object kind or featural information to individuate items (Van de Walle, Carey, \& Prevor, 2000). At 3 years of age, children seem predisposed to consider disconnected objects as the basic unit for numerosity judgments, even when the task instructions specifically ask for the number of kinds or features (Shipley \& Shepperson, 1990). However, a subsequent study determined that 3 -year-olds can in fact individuate and count whole objects, parts, and holes (Giralt \& Bloom, 2000). This literature suggests that throughout development infants gain the ability to individuate items based on an increasing number of levels of specificity, from discrete objects based on spatiotemporal and connectivity at a few months of age, to the ability to individuate parts of a single object at 3 years. The question remains, however, do whole physically separated objects maintain a special status throughout adulthood as they do in early infancy? The experiments discussed above do not allow us to discriminate between serial or parallel processing; thus the question remains, can adults individuate connected object parts in parallel?

Objects and object parts can be discriminated based on connectivity; the effect of connectivity on individuation has been studied in both MOT and estimation paradigms. In MOT, when target stimuli are perceived as connected to a distracting element, performance is significantly worse than when compared to tracking of spatially disconnected targets (Scholl, Pylyshyn, \& Feldman, 2001). The exception in Scholl et al. (2001) was a condition where the target and distractors were connected by lines that criss-crossed in a random fashion resulting in the perception of two items connected by a substance. Substances cannot be efficiently tracked (vanMarle \& Scholl, 2003). Thus connectivity seems to interfere with object tracking except for when the connecting element is one that cannot be tracked, and as such allows for the isolated individuation of one part of the stimulus. Franconeri, Bemis, and Alvarez (2009) found that adding task irrelevant connecting lines between target shapes caused subjects to underestimate the number of targets, suggesting that estimation relies on representations of distinct unconnected objects. A convergent study suggested that connectivity affects the initial individuation of targets to be estimated, rather than influencing the subsequent magnitude judgment (He, Zhang, Zhou, \& Chen, 2009). If estimation relies on the same individuation mechanism as observed in subitizing, we would expect that in a rapid enumeration paradigm subjects would underestimate and thus perform poorly when enumerating object parts.

While connectivity has not been thoroughly tested in the context of rapid enumeration, previous work suggests that subitizing seems to occur over spatially separate, disconnected objects, and not features (Trick \& Pylyshyn, 1994; Watson \& Maylor, 2006). Parallel individuation does not function over features such as color or orientation, unless the items are grouped into distinct locations (Watson Maylor, \& Bruce, 2005). While featural information does not seem to be a minimum requirement for object individuation, and distinct locations are emphasized as important in theories that take into account the developmental trend of individuation ability (Leslie et al., 1998) as well as in MOT (vanMarle \& Scholl, 2003), location and "objecthood" remain conflated within the context of subitizing. Object features or parts can be spatially distinct in that a cup handle, or two handles on a child's cup, can exist in different locations while being part of the same object. Is objecthood critical to parallel individuation? Or is it sufficient that items, either connected parts or whole physically separate objects, occupy distinct locations in space? 
Using the subitizing phenomenon to measure parallel individuation, we explored whether subjects showed evidence of a subitizing "elbow" when enumerating object features, such as object parts. In Experiment 1, we tested whether subitization occurs over protrusions on a single circle. We then added irregularity to the protrusions in Experiment 2 to test the generalization of individuation over different shapes. To reduce the possibility that the parts were viewed as overlapping physically separated objects, we decreased the separability of the protrusions in Experiment 3, and removed any t-junction occlusion cue that might aid individuation in Experiment 4. We tested the performance of subjects with limited presentation time in Experiment 5 by measuring error rate. Lastly, in Experiment 6, we used line stimuli to eliminate any perception of occlusion in our single-object displays. If the individuation mechanism that allows for the simultaneous selection of things-out-there functions over distinct locations without requiring "objecthood," we would expect to see subitization of stimulus parts.

For each experiment, we aimed for 12 to 17 participants, based on a required sample size estimate of 12 , computed for a difference between paired means using G*Power software (Faul, Erdfelder, Lang, \& Buchner, 2007) with $\alpha=.05$, and power set to .8. Given the prevalence of the subitizing effect in the literature, we expected a large effect, and chose an effect size of 0.8 according to Cohen's effect size index (Cohen, 1988). Variability in the sample size of each experiment reflects the variability of available subjects in the subject pool.

\section{Experiment 1: Single-object spike protrusions and multi-object displays}

\section{Introduction}

In Experiment 1, we tested whether individuation functions over connected stimulus parts, as well as over physically separate objects. To do this, we asked participants to perform an enumeration task for both protrusions and objects.

\section{Method}

Participants Fourteen Harvard University students participated for compensation of either US $\$ 8$ or course credit. All participants provided informed consent, as approved by the Committee on the Use of Human Subjects in Research at Harvard University. All participants were debriefed about the purpose of the study and supplied with supplemental reading after completion of the experiment.

Stimuli Displays consisted of two types of stimuli: (1) multiobject displays consisting of 1-8 physically separate objects as typically used in subitizing tasks, and (2) single-object displays consisting of a single object with 1-8 connected target features to be enumerated. Stimuli were created in Adobe Illustrator. Singleobject displays consisted of black target shapes overlapping a black circle. The central circle was selected pseudorandomly from two sizes for each display to avoid the overall area correlating directly with the number of protruding shapes. The target shapes or features were selected from two sizes of spikes. Multiobject displays consisted of spatially separate black dots, positioned at the locations corresponding to the ends of the spikes in the test displays to match for location and eccentricity. Four different variants of a display were created for each stimulus type and number. Stimuli were presented against a gray background. All subjects performed the experiment on the same computer and in the same testing room. Visual stimuli subtended a maximum of $14^{\circ}$ visual angle, and were presented using Matlab with the Psychophysics Toolbox extensions (Brainard, 1997; Kleiner, Brainard, \& Pelli, 2007; Pelli, 1997).

Procedure Subjects received instructions to report the number of circles or spikes they saw on the screen as quickly and as accurately as possible. Subjects were asked to state their response out loud while pressing the space bar to record their reaction time (Watson et al., 2005). This ensured they would not use an alternate strategy that might bias their response times, such as reliance on after-images. After indicating their response verbally and via space bar press, subjects recorded their response via the number keys on the keyboard. In written and verbal instructions, we avoided using terminology such as "counting" to avoid biasing the subjects' enumeration strategy and instead asked subjects to report "how many" target items were present.

Subjects completed a practice round consisting of two blocks, each consisting of 32 presentations of one display type (multi-object or single-object). The practice round was followed by the full-length experiment consisting of 160 presentations per block ( 4 variants $\times 5$ presentations $\times 8$ numerosities). Each block contained displays of the same display type, and the order of the blocks was counterbalanced across subjects. The order of the displays within a block was determined pseudorandomly, constrained such that no two identical displays were presented in succession. Each trial consisted of: $1 \mathrm{~s}$ fixation, display presentation for $4 \mathrm{~s}$ or until the participant pressed the space bar indicating a response, fixation until number key pressed (only possible answers accepted), and $1 \mathrm{~s}$ blank. Every 40 trials, the participants were given the option to take a self-timed break before continuing.

Analyses Incorrect trials, as well as trials more than two standard deviations from each subject's mean correct response reaction time for each numerosity, were removed from analysis (average: $9.4 \%$ of trials). For all subjects, trials for the highest numerosity were discarded to avoid end effects (Trick \& Pylyshyn, 1993). 
First we tested for the presence of a subitizing elbow in each display type by comparing the reaction time slopes in the subitizing range and the counting range for each individual. The location of the split between the counting and subitizing ranges was determined using a piecewise linear model from the R library SiZer (Sonderegger, 2012). This models two lines and outputs the slopes and break point between the two lines that best fit the data (see Ester, Drew, Klee, Vogel, \& Awh, 2012; Pagano, Lombardi, \& Mazza, 2014 for similar analyses). The differences in slopes between individual best-fit counting and subitizing ranges for each display type were entered into one-tailed paired t-tests; the expectation was that compared to the subitizing range the counting slope would be greater, and the difference therefore significantly positive. For these one-tailed paired t-tests we report two-tailed $90 \%$ confidence intervals (CIs) in our results tables, as the lower bound of a two-tailed $90 \% \mathrm{CI}$ is mathematically equivalent to a single-tailed $95 \% \mathrm{CI}$.

To ensure that a bilinear model actually best reflected the trends in our data, we compared the adjusted $\mathrm{R}^{2}$ from the piecewise linear model to the adjusted $\mathrm{R}^{2}$ of an exponential model (see Balakrishnan \& Ashby, 1991) using a two-tailed paired t-test. The modeling was performed following the method of Pagano, Lombardi, \& Mazza (2014). The piecewise linear modeling was performed using the R library SiZer (Sonderegger, 2012), and the exponential modeling was performed with a customized R script, derived from the Nelder-Mead optimization algorithm (Nelder \& Mead, 1965), that allowed two free parameters to be estimated for each subject: $R T=\beta e^{\alpha n}$ or error $=\beta^{\alpha n}$ depending on dependent measure of interest, where $e$ is the base of the natural logarithm, $\mathrm{n}$ is set numerosity, and the two estimated parameters are $\alpha$ and $\beta$.

To compare trends in performance across the two display types, we tested the difference between slopes for each of the counting and subitizing ranges with two-tailed paired t-tests. We also tested the difference in subitizing ranges (break points) between the two display types with two-tailed paired t-tests.

For all tests, we calculated two effect sizes: Cohen's $d$ for a paired design $\left(d=\frac{M_{d i f}}{S_{a v}}\right)$, and an unbiased estimate of Cohen's $\mathrm{d}\left(d_{u n b}=\left(1-\frac{3}{4 d f-1}\right) \times d\right)($ Cummings, 2012).

\section{Results}

We first tested for evidence of subitization by comparing slopes between the counting and subitizing ranges (see Table 1 for all Experiment 1 statistics.) We observed the subitizing elbow, represented by significantly higher counting slopes than subitizing slopes, in both the multi-object and the single-object displays. The mean difference in slopes for both conditions had large effect sizes greater than 2 . We also tested that a bilinear model, demonstrating the presence of subitizing in the lower numerosities and lack of subitizing in higher numerosities, would best fit the data. While both the bilinear (multi-object: mean adjusted $\mathrm{R}^{2}=.99$, singleobject: mean adjusted $\mathrm{R}^{2}=.98$ ) and exponential (multiobject: mean adjusted $\mathrm{R}^{2}=.89$, single-object: mean adjusted $\mathrm{R}^{2}=.91$ ) models fit the data fairly well, a statistical comparison showed that the bilinear model better accounted for the data observed with effect sizes over 1 for both display types.

We then investigated the effects of display type on performance. The average best-fit subitizing range for the single-object displays was 3.68 spikes, and 3.85 circles for the multi-object displays. There was no significant difference and a small effect size for the mean difference in subitizing range across the two display types. There was also no significant difference in slope across display types for either the subitizing range or the counting range. See Table 2 for slopes for each condition, and Fig. 1 for RT and error rates.

\section{Discussion}

These results demonstrate that subitizing occurs over object parts, such as spikes on a circle.

\section{Experiment 2: Single-object varied protrusions and multi-object displays}

\section{Introduction}

To control for the possibility that the single feature "spikiness" could be easily selected and used to aid individuation, we varied the shapes of the protrusions.

\section{Method}

Participants Thirteen Harvard University students participated for compensation of either US\$8 or course credit. All participants provided informed consent, as approved by the Committee on the Use of Human Subjects in Research at Harvard University. All participants were debriefed about the purpose of the study and supplied with supplemental reading after completion of the experiment.

Stimuli In this experiment, we varied the shape of the singleobject features ("protrusions") to control for the possibility that a single feature such as spikiness can aid selection early 
Table 1 Statistical results from Experiment 1

Experiment 1

df T-value $p$-value Mean difference SD

CI

Cohen's D d-unb

Presence of Subitizing

Subitizing Elbow: Difference in slope (Counting-Subitizing)

Multi-object

$13 \quad 6.56<.001 \quad 309.44$

$90 \% \mathrm{CI}$

Single-object

$13 \quad 7.00<.001 \quad 226.34 \mathrm{~ms}$

Best Fit Model: Bilinear-Exponential R2-adjusted

Multi-object

$\begin{array}{llll}13 & 2.89 & .013 & 0.10\end{array}$

$\begin{array}{llll}13 & 3.71 \quad .003 & 0.07\end{array}$

0.07

Single-object

Effects of Display Type:

Difference in subitizing range (Multi-object-Single-object)

Subitizing range

$13 \quad 0.69>250 \quad 0.17$ items

$176.40 \quad[225.95-392.93] \quad 2.87$

2.71

Difference in slope (Multi-object-Single-object)

Subitizing

Counting

$\begin{array}{llll}13 & -2.11 & .055 & -28.81 \mathrm{~ms} \\ 13 & 1.48 & .164 & 54.29 \mathrm{~ms}\end{array}$

$120.98 \quad[169.08-283.60] \quad 2.33$

$$
95 \% \mathrm{CI}
$$

$\begin{array}{llll}0.13 \quad[0.02-0.17] & 1.08 & 1.01\end{array}$

$0.07 \quad[0.03-0.11] \quad 1.30 \quad 1.23$

Note: Statistical results testing the presence of subitizing and effects of display type. For the one-tailed t-tests examining the presence of a subitizing elbow, we report a two-tailed $90 \%$ CI. For all other tests we report a $95 \%$ CI. See analyses of Experiment 1 for more details. $d$-unb d-unbiased, as calculated from Cohen's-d

in processing, in contrast to the curvature of the central object (Fig. 1). The stimuli were thus created in the same manner as Experiment 1, with the exception that the target shapes were selected from ten shapes of varied form. Multi-object displays were created by removing the central circle and slightly rotating the protruding shapes to avoid the percept of a circular illusory contour. Four different variants of a display were created for each stimulus type and number. Visual stimuli subtended a maximum of $11.5^{\circ}$ visual angle. Presentation of the stimuli was identical to Experiment 1.

Procedure The same procedure was used as in Experiment 1, except subjects were now instructed to report the number of shapes or protrusions they saw on the screen as quickly and accurately as possible.

Analyses The same data trimming procedure (average: 7.2 \% of trials) and analyses were used as in Experiment 1.

\section{Results}

We first tested for evidence of subitizing by comparing slopes between the counting and subitizing ranges (see Table 3 for all Experiment 2 statistics). We observed the subitizing elbow, represented by significantly higher counting slopes than subitizing slopes, in both the multi-object and single-object displays. The mean difference in slopes for both conditions had large effect sizes greater than 2.5. We also tested that a bilinear model, demonstrating the presence of subitizing in the lower numerosities and lack of subitizing in higher numerosities, would best fit the data. While both the bilinear (multi-object: mean adjusted $\mathrm{R}^{2}=.95$, singleobject: mean adjusted $\mathrm{R}^{2}=.94$ ) and exponential (multi-

Table 2 Average subitizing and counting slopes

\begin{tabular}{llc}
\hline Average individual slopes & $\begin{array}{c}\text { Subitizing } \\
\text { Slope }\end{array}$ & $\begin{array}{c}\text { Counting } \\
\text { Slope }\end{array}$ \\
\cline { 2 - 3 } & $\begin{array}{ll}\text { (individual) } \\
\text { (individual) }\end{array}$ \\
\hline (Measure of interest: RT) & $9.4 \mathrm{~ms}$ & $318.8 \mathrm{~ms}$ \\
Exp. 1 MO: Circles & $38.2 \mathrm{~ms}$ & $264.6 \mathrm{~ms}$ \\
Exp. 1 SO: Spikes & $28.0 \mathrm{~ms}$ & $270.2 \mathrm{~ms}$ \\
Exp. 2 MO: Shapes & $36.6 \mathrm{~ms}$ & $237.5 \mathrm{~ms}$ \\
Exp. 2 SO: Protrusions & $42.4 \mathrm{~ms}$ & $290.4 \mathrm{~ms}$ \\
Exp. 3 SO: Outdents & $55.7 \mathrm{~ms}$ & $312.2 \mathrm{~ms}$ \\
Exp. 3 SO: Indents & $5.3 \mathrm{~ms}$ & $305.3 \mathrm{~ms}$ \\
Exp. 4 MO: Shapes & $50.3 \mathrm{~ms}$ & $234.9 \mathrm{~ms}$ \\
Exp. 4 SO: Protrusions & & \\
(Measure of Interest: Error Rate) & $1.4 \%$ & $16.0 \%$ \\
Exp. 5 MO: Shapes & $1.5 \%$ & $19.3 \%$ \\
Exp. 5 SO: Protrusions & & \\
(Measure of Interest: RT) & $13.3 \mathrm{~ms}$ & $246.0 \mathrm{~ms}$ \\
Exp. 6 MO: Outline Shapes & $55.0 \mathrm{~ms}$ & $268.3 \mathrm{~ms}$ \\
Exp. 6 SO: Outline & & \\
$\quad$ Protrusions & & \\
\hline
\end{tabular}

Note: Average individual slopes for the subitizing and counting ranges as determined by the bilinear model. For Experiments 1-4 and Experiment 6, the slope reflects the increase in RT in ms for each additional item enumerated. For Experiment 5, the slope reflects the increase in error rate for each additional item enumerated

$R T$ reaction time, $M O$ multi-object display, $S O$ single-object display 


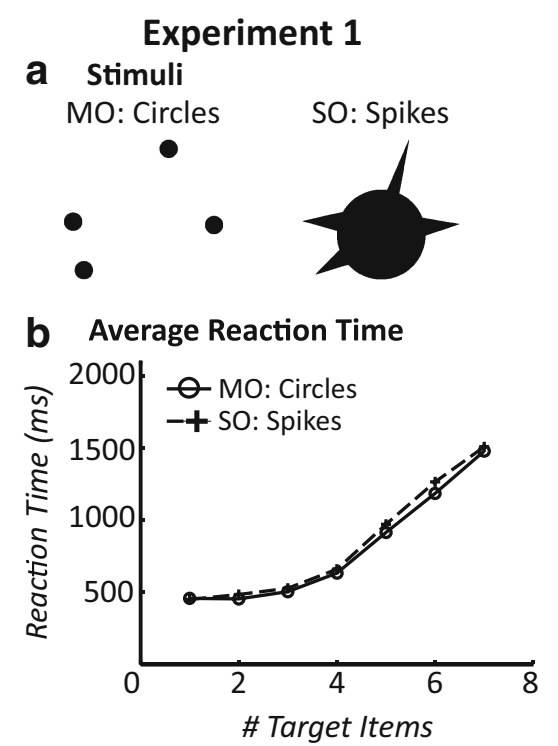

C Average Error Rate

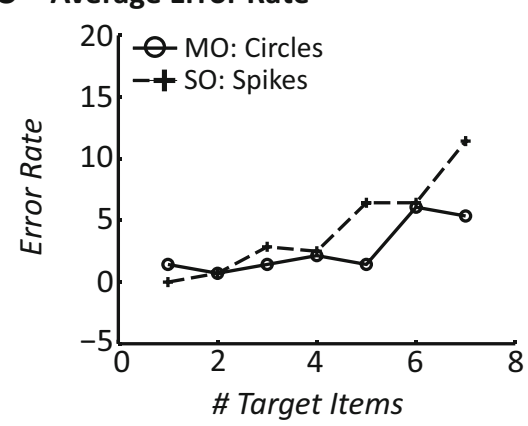

Fig. 1 Stimuli, average reaction times and error rates for Experiments 1 and 2. Results for Experiments 1 and 2 showing (a) example stimuli, (b) average reaction times, and (c) average error rates. Average reaction time

object: mean adjusted $\mathrm{R}^{2}=.90$, single-object: mean adjusted $\mathrm{R}^{2}=.90$ ) models fit the data fairly well, a statistical comparison showed that the bilinear model better accounted for the data observed with effect sizes over 1 for both display types.

We then investigated the effects of display type on performance. The average best-fit subitizing range for the singleobject displays was 3.74 protrusions, and 3.88 shapes for the multi-object displays. There was no significant difference and a small effect size for the mean difference in subitizing range across the two display types. There was also no significant difference in slope across display types for either the subitizing range or the counting range (see Table 2 for slopes for each condition, and Fig. 1 for reaction time and error rates).

\section{Discussion}

Despite the varied shapes of the protrusions, we observed subitization in both display types. This suggests the behavior

\section{Experiment 2}
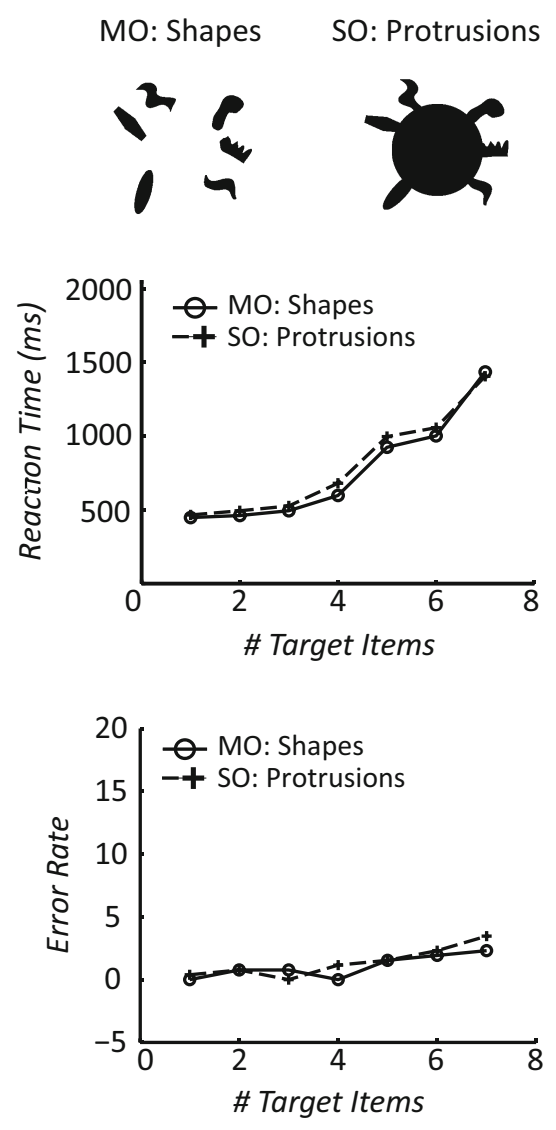

data is reflected in ms, and average error rates are reflected in percent. $M O$ multi-object display, $S O$ single-object display

observed does not result from one simple feature ("spikiness") aiding selection of the object parts.

\section{Experiment 3: Single-object indent and outdent displays}

\section{Introduction}

To explore whether the previous data resulted from participants viewing the parts as separable objects occluding the central circle, we decreased the separability of the parts in two single-object displays. We reduced the size of the protrusions in "outdent" displays, and created features intrinsic to the object in "indent" displays.

\section{Method}

Participants Twelve Harvard University students participated for compensation of either US $\$ 8$ or course credit. All 
Table 3 Statistical results from Experiment 2

\begin{tabular}{|c|c|c|c|c|c|c|c|c|}
\hline Experiment 2 & df & T-value & p-value & Mean difference & SD & $\mathrm{CI}$ & Cohen's D & d-unb \\
\hline \multicolumn{9}{|l|}{ Presence of Subitizing: } \\
\hline Subitizing Elbow: Difference in slope (Counting-Subitizing) & & & & & & $90 \% \mathrm{CI}$ & & \\
\hline Multi-Object & 12 & 7.73 & $<.001$ & $242.25 \mathrm{~ms}$ & 112.95 & [186.42-298.08] & 2.76 & 2.59 \\
\hline Single-Object & 12 & 8.95 & $<.001$ & $200.88 \mathrm{~ms}$ & 80.91 & [160.88-240.87] & 2.97 & 2.78 \\
\hline Best Fit Model: Bilinear-Exponential R2-adjusted & & & & & & $95 \% \mathrm{CI}$ & & \\
\hline Multi-Object & 12 & 2.83 & .015 & 0.05 & 0.07 & {$[0.01-0.09]$} & 1.29 & 1.21 \\
\hline Single-Object & 12 & 4.90 & $<.001$ & 0.04 & 0.03 & {$[0.02-0.06]$} & 1.31 & 1.22 \\
\hline \multicolumn{9}{|l|}{ Effects of Display Type: } \\
\hline Difference in subitizing range (Multi-object-Single-object) & & & & & & $95 \% \mathrm{CI}$ & & \\
\hline Subitizing Range & 12 & 0.50 & $>.250$ & 0.14 items & 1.02 & {$[-0.47-0.76]$} & 0.14 & 0.13 \\
\hline Difference in slope (Multi-object-Single-object) & & & & & & $95 \% \mathrm{CI}$ & & \\
\hline Subitizing & 12 & -1.04 & $>.250$ & $-8.64 \mathrm{~ms}$ & 29.87 & {$[-26.70-9.41]$} & -0.31 & -0.29 \\
\hline Counting & 12 & 1.16 & $>.250$ & $32.73 \mathrm{~ms}$ & 102.15 & {$[-29.00-94.46]$} & 0.31 & 0.29 \\
\hline
\end{tabular}

Note: Statistical results testing the presence of subitizing and effects of display type. For the one-tailed t-tests examining the presence of a subitizing elbow, we report a two-tailed $90 \%$ CI. For all other tests we report a $95 \%$ CI. See analyses of Experiment 1 for more details. $d$-unb d-unbiased, as calculated from Cohen's-d

participants provided informed consent, as approved by the Committee on the Use of Human Subjects in Research at Harvard University. All participants were debriefed about the purpose of the study and supplied with supplemental reading after completion of the experiment.

Stimuli Two types of single-object displays were created to reduce the possibility of participants viewing the parts as separable objects occluding the central circle (Fig. 2). The displays consisted of black circles overlapped with either black semicircle "outdents" that were smaller and more difficult to separate from the central stimuli, or gray semicircle "indents" intrinsic to the central object and the same color as the background. The locations of the single-object display target features were generated randomly for each stimulus and each participant, constrained such that no two features could overlap. The stimuli subtended a maximum of $7.5^{\circ}$ visual angle and were created in Matlab using the Psychophysics Toolbox extensions (Brainard, 1997; Kleiner et al, 2007; Pelli, 1997).

Procedure The same procedure as in Experiment 1 was used, with the exception of a change in instruction and experiment length. Subjects were asked to report how many indents or outdents were in each display, and each block consisted of 240 trials (30 variants $\times 8$ numerosities). Since participants could have still viewed the indent condition as small physically separate objects overlapping the larger circle we controlled for this confound by asking each participant after the experiment how they perceived the indent condition. They were provided with the following options with verbal descriptions and example drawings: (a) bites out of a cookie, (b) half moons overlapping a circle, (c) small circles overlapping a large circle, or (d) other.

Analyses The same analyses were performed as in Experiments 1 and 2. The average percent of trials removed in trimming per numerosity was $6.6 \%$.

\section{Results}

We first tested for evidence of subitizing by comparing slopes between the counting and subitizing ranges (see Table 4 for all Experiment 3 statistics). We observed the subitizing elbow, represented by significantly higher counting slopes than subitizing slopes, in both variants of the single-object displays (indents and outdents). The mean difference in slopes for both conditions had large effect sizes greater than 2. As in Experiments 1 and 2, both the bilinear (indent: mean adjusted $\mathrm{R}^{2}=.98$, outdent: mean adjusted $\mathrm{R}^{2}=.99$ ) and exponential (indent: mean adjusted $\mathrm{R}^{2}=.94$, outdent: mean adjusted $\left.\mathrm{R}^{2}=.95\right)$ models fit the data well. However, the bilinear model better accounted for the data observed for both testing conditions, with effect sizes greater than 1 for both display types.

We then investigated the effects of display type on performance. The average best-fit subitizing range for each display 
Experiment 3

a Stimuli

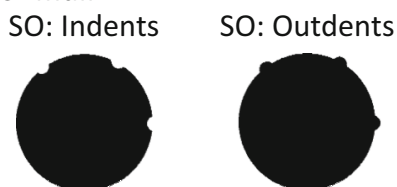

b Average Reaction Time

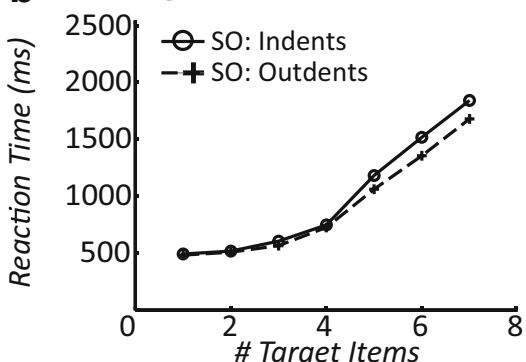

C Average Error Rate

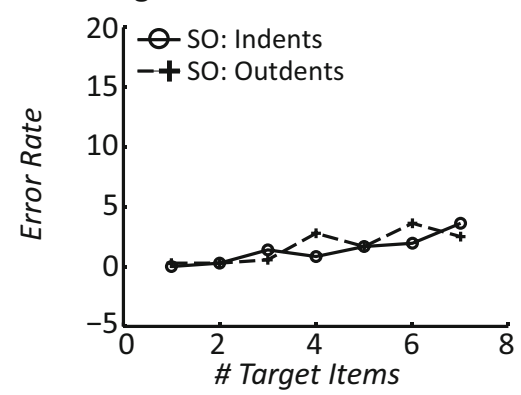

Fig. 2 Stimuli, average reaction times and error rates for Experiments 3 and 4. Results for Experiments 3 and 4 showing (a) example stimuli, (b) average reaction times, and (c) average error rates. Average reaction time

type was 3.67 outdents, and 3.59 indents. There was no significant difference and a small effect size for the mean difference in subitizing range across the two display types. There was also no significant difference in slope across display types for either the subitizing range or the counting range. See Table 2 for slopes for each condition, and Fig. 2 for reaction time and error rates.

In response to our poll about how participants viewed the indent condition, 8 of the 12 participants responded "bites out of a cookie" or "valleys." One participant was contacted via email 6 days after completing the task, and did not view the drawings. However, the participant responded with an unprompted vivid description, so it seems the drawings were not needed in that case. To test whether the four participants who perceived the indents as separable objects affected the results, we carried out the analyses using only the eight subjects who perceived the indents as "bites." No difference was observed in the results.

\section{Experiment 4}
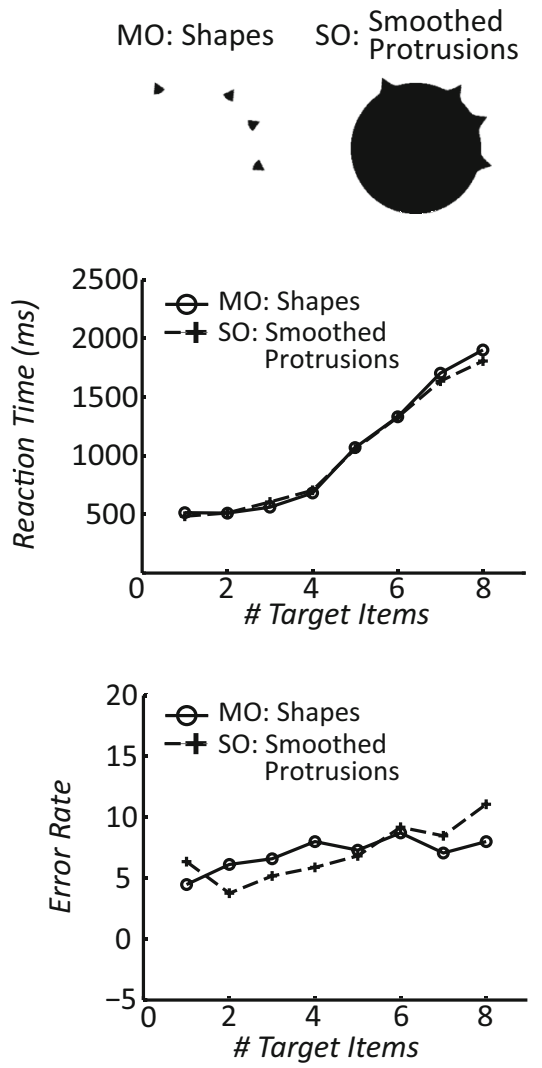

data is reflected in ms, and average error rates are reflected in percent. $M O$ multi-object display, $S O$ single-object display

\section{Discussion}

Subitization occurred for both indents and outdents, suggesting that the individuation mechanism can function over stimuli difficult to separate, or intrinsic to, the central object.

\section{Experiment 4: Single-object smoothed protrusions and multi-object displays}

\section{Introduction}

While Experiment 3 decreased the separability of the object parts, it did not eliminate possible cues for occlusion. The presence of t-junctions along a contour is a major cue when determining whether an image is one object or multiple objects with partial occlusion (Rubin, 
Table 4 Statistical results from Experiment 3

\begin{tabular}{|c|c|c|c|c|c|c|c|c|}
\hline Experiment 3 & df & T-value & p-value & Mean difference & SD & $\mathrm{CI}$ & Cohen's D & d-unb \\
\hline \multicolumn{9}{|l|}{ Presence of Subitizing: } \\
\hline Subitizing Elbow: Difference in slope (Counting-Subitizing) & & & & & & $90 \% \mathrm{CI}$ & & \\
\hline Indent & 11 & 6.74 & $<.001$ & $256.47 \mathrm{~ms}$ & 113.87 & {$[188.10-324.83]$} & 2.57 & 2.39 \\
\hline Outdent & 11 & 9.62 & $<.001$ & $247.92 \mathrm{~ms}$ & 89.24 & [201.65-294.18] & 4.04 & 3.76 \\
\hline Best Fit Model: Bilinear-Exponential R2-adjusted & & & & & & $95 \% \mathrm{CI}$ & & \\
\hline Indent & 11 & 5.07 & $<.001$ & 0.04 & 0.03 & {$[0.02-0.06]$} & 1.61 & 1.50 \\
\hline Outdent & 11 & 5.30 & $<.001$ & 0.04 & 0.02 & {$[0.02-0.05]$} & 1.57 & 1.46 \\
\hline \multicolumn{9}{|l|}{ Effects of Display Type: } \\
\hline Difference in subitizing range (Indents-Outdents) & & & & & & $95 \% \mathrm{CI}$ & & \\
\hline Subitizing Range & 11 & -0.58 & $>.250$ & -0.08 items & 0.46 & {$[-0.37-0.22]$} & -0.12 & -0.11 \\
\hline Difference in slope (Indents-Outdents) & & & & & & $95 \% \mathrm{CI}$ & & \\
\hline Subitizing & 11 & 1.25 & .239 & $13.25 \mathrm{~ms}$ & 36.86 & {$[-10.17-36.67]$} & 0.50 & 0.46 \\
\hline Counting & 11 & 1.00 & $>.250$ & $21.80 \mathrm{~ms}$ & 75.67 & {$[-26.27-69.88]$} & 0.19 & 0.18 \\
\hline
\end{tabular}

Note: Statistical results testing the presence of subitizing and effects of display type. For the one-tailed t-tests examining the presence of a subitizing elbow, we report a two-tailed $90 \%$ CI. For all other tests we report a $95 \%$ CI. See analyses of Experiment 1 for more details. $d$-unb d-unbiased, as calculated from Cohen's-d

2001). Here, we created protrusions with smoothed contours to eliminate this cue.

\section{Method}

Participants Nineteen Harvard University students participated for compensation of either US\$8 or course credit. All participants provided informed consent, as approved by the Committee on the Use of Human Subjects in Research at Harvard University. All participants were debriefed about the purpose of the study and supplied with supplemental reading after completion of the experiment. Two participants were excluded for poor task performance with more than $30 \%$ of trials from multiple numerosities removed according to the trimming procedure described in the method for Experiment 1.

Stimuli In order to avoid participants viewing the single-object displays as partially occluding shapes, we removed any tjunctions from the stimuli. Single-object displays consisted of a black circle with overlapping arcs, and the junctions between the arcs and the central circle were smoothed to form a continuous contour. Multi-object displays consisted of the arcs with the central circle removed (Fig. 2). The locations of the singleobject display target features were generated randomly for each stimulus and each participant, constrained such that no two features could overlap. The arc orientation in the multi-object displays was randomly selected from a set of angles $\left(-20^{\circ}\right.$, $\left.-10^{\circ}, 0^{\circ},+10^{\circ},+20^{\circ}\right)$ to avoid the illusory percept of a circle. Visual stimuli subtended a maximum of $9^{\circ}$ visual angle, and were presented in the same manner as Experiment 3.

Procedure The same procedure as in Experiment 1 was used, with the exception of a change in instruction and experiment length. Subjects were asked to report how many protrusions or shapes were in each display, and each block consisted of 215 trials $(25$ variants $\times 8$ numerosities, 15 variants $\times 9$ th numerosity).

Analyses The same analyses were performed as in Experiments 1 and 2. The average percent of trials removed in trimming per numerosity was $13.5 \%$.

Results We observed the subitizing elbow, represented by significantly higher counting slopes than subitizing slopes, in both the multi-object and the single-object displays (see Table 5). The mean difference in slopes for both conditions had large effect sizes greater than 2.5. We also tested that a bilinear model, demonstrating the presence of subitizing in the lower numerosities and lack of subitizing in higher numerosities, would best fit the data. While both the bilinear (multiple object: mean adjusted $\mathrm{R}^{2}=.97$, single object: mean 
Table 5 Statistical results from Experiment 4

\begin{tabular}{|c|c|c|c|c|c|c|c|c|}
\hline Experiment 4 & $\mathrm{df}$ & T-value & p-value & Mean difference & SD & $\mathrm{CI}$ & Cohen's D & d-unb \\
\hline \multicolumn{9}{|l|}{ Presence of Subitizing: } \\
\hline Subitizing Elbow: Difference in slope (Counting-Subitizing) & & & & & & $90 \% \mathrm{CI}$ & & \\
\hline Multi-Object & 16 & 10.84 & $<.001$ & $299.94 \mathrm{~ms}$ & 114.13 & [251.62-348.27] & 4.25 & 4.05 \\
\hline Single-Object & 16 & 7.47 & $<.001$ & $184.61 \mathrm{~ms}$ & 101.90 & {$[141.46-227.75]$} & 2.93 & 2.79 \\
\hline Best Fit Model: Bilinear-Exponential R2-adjusted & & & & & & $95 \% \mathrm{CI}$ & & \\
\hline Multi-Object & 16 & 9.66 & $<.001$ & 0.04 & 0.02 & {$[0.03-0.05]$} & 1.29 & 1.23 \\
\hline Single-Object & 16 & 6.89 & $<.001$ & 0.03 & 0.02 & {$[0.02-0.04]$} & 0.47 & 0.45 \\
\hline \multicolumn{9}{|l|}{ Effects of Display Type: } \\
\hline Difference in subitizing range (Multi-object-Single-object) & & & & & & $95 \% \mathrm{CI}$ & & \\
\hline Subitizing Range & 16 & -1.10 & $>.250$ & -0.22 items & 0.81 & {$[-0.63-0.20]$} & -0.28 & -0.26 \\
\hline Difference in slope (Multi-object - single-object) & & & & & & $95 \% \mathrm{CI}$ & & \\
\hline Subitizing & 16 & -2.82 & .012 & $-44.99 \mathrm{~ms}$ & 65.82 & {$[-78.83--11.15]$} & -1.03 & -0.98 \\
\hline Counting & 16 & 3.53 & .003 & $70.35 \mathrm{~ms}$ & 82.18 & [28.10-112.60] & 0.84 & 0.80 \\
\hline
\end{tabular}

Note: Statistical results testing the presence of subitizing and effects of display type. For the one-tailed t-tests examining the presence of a subitizing elbow, we report a two-tailed $90 \%$ CI. For all other tests we report a $95 \%$ CI. See analyses of Experiment 1 for more details. $d$-unb d-unbiased, as calculated from Cohen's-d

adjusted $\mathrm{R}^{2}=.96$ ) and exponential (multiple object: mean adjusted $\mathrm{R}^{2}=.93$, single object: mean adjusted $\mathrm{R}^{2}=.92$ ) models fit the data fairly well, a statistical comparison showed that the bilinear model better accounted for the data observed with a large effect size for the multi-object displays. The bilinear model also showed statistically better performance for single-object displays, but with a small effect size.

We then investigated the effects of display type on performance. The average best-fit subitizing range for the singleobject displays was 3.58 smoothed protrusions, and 3.37 shapes for the multi-object displays. There was no significant difference and a small effect size for the mean difference in subitizing range across the two display types. The difference in slopes across display types was significant in both the subitizing range and the counting range. See Table 2 for slopes for each condition, and Fig. 2 for reaction time and error rates.

Discussion To remove occlusion cues from the stimuli, we smoothed the junctions between the central object and the protrusions. While there were significant differences in slope across display types, the presence of a bilinear elbow supports subitizing in both cases. While these results support parallel individuation of both stimulus types, it is possible that the differences in slope observed reflect a less efficient individuation of the smoothed protrusions than the shapes. Nonetheless, these data together with Experiment 3 suggest the participants did not view the protrusions as physically separate objects, but instead can individuate object parts without visual cues supporting separability.

\section{Experiment 5: Speeded presentation of single-object and multi-object displays}

\section{Introduction}

The long presentation in previous experiments allowed subjects to accurately count the higher numerosities. To test for subitizing under more pressured conditions, we forced estimation by limiting the presentation time to $200 \mathrm{~ms}$.

\section{Method}

Participants Thirteen Harvard University students participated for compensation of either US\$8 or course credit. One participant was removed from analysis for accuracy below $80 \%$ for all numerosities. All participants provided informed consent, as approved by the Committee on the Use of Human Subjects in Research at Harvard University. All participants were debriefed about the purpose of the study and supplied with supplemental reading after completion of the experiment.

Stimuli The same stimuli as described in Experiment 4 were used.

Procedure The same procedure was used as in Experiment 4, except instructions only emphasized accuracy and not speed since the presentation time of the stimuli was fixed at $200 \mathrm{~ms}$. Subjects recorded how many items they believed were in each display via keypress after each stimulus presentation. Each 
block consisted of 250 trials (30 variants $\times 8$ numerosities, 10 variants $\times 9$ th numerosity).

Analyses The analyses performed were the same as described in Experiment 1, with one exception: instead of testing for the presence of a subitizing elbow in reaction times, we compared slopes and subitizing ranges for the error rates of each display type. ${ }^{1}$

Results We first tested for evidence of subitization by comparing error rate slopes between the counting and subitizing ranges (see Table 6 for all Experiment 5 statistics). We observed the subitizing elbow, represented by significantly higher counting slopes than subitizing slopes, in both the multi-object and the single-object displays. The mean difference in slopes for both conditions had large effect sizes greater than 1 . We also tested that a bilinear model, demonstrating the presence of subitizing in the lower numerosities and lack of subitizing in higher numerosities, would best fit the data. While the bilinear model fit the data moderately well for the multiple object displays (mean adjusted $\mathrm{R}^{2}=.76$ ) and quite well for the single object displays (mean adjusted $\mathrm{R}^{2}=.93$ ) the exponential model performed moderately worse across both display types (multiple object: mean adjusted $\mathrm{R}^{2}=.67$, single object: mean adjusted $\mathrm{R}^{2}=.88$ ). A statistical comparison showed that the bilinear model better accounted for the data observed with effect sizes over .3 for both display types.

We then investigated the effects of display type on performance. The average best-fit subitizing range for the singleobject displays was 5.18 spikes, and 5.47 circles for the multi-object displays. There was no significant difference and a small effect size for the mean difference in subitizing range across the two display types. There was also no significant difference in slope across display types for either the subitizing range or the counting range. See Table 2 for slopes for each condition, and Fig. 3 for plotted error rates.

Discussion We explored subitization performance when presentation time was limited so high accuracy for large numerosities was impossible. We saw evidence of subitizing in error rates for both display types, without significant differences in slopes or subitizing range across types. Interestingly,

\footnotetext{
${ }^{1}$ While analyzing error rates is typical in the subitizing literature, in the case of short presentation times we can also investigate differences in display type based on underestimation in average responses. We saw no underestimation or differences in average responses across conditions until the counting range, which is consistent with our findings in Table 6 investigating effects of display type. See Appendix for graph.
}

the subitizing ranges for both display types were larger than those observed in the previous experiments, which may result from participants performing at ceiling for the lower numerosities.

\section{Experiment 6: Single-object and multi-object outline displays}

\section{Introduction}

To eliminate any perception of the protruding targets in our single-object display as multiple objects occluding a central circle, we performed an additional experiment using line stimuli. (See Fig. 4.)

\section{Method}

Participants Twelve Harvard University students participated for compensation of either US\$8 or course credit. All participants provided informed consent, as approved by the Committee on the Use of Human Subjects in Research at Harvard University. All participants were debriefed about the purpose of the study and supplied with supplemental reading after completion of the experiment.

Stimuli Single-object displays consisted of a black outline of a circle with protruding arcs. Multi-object displays consisted of outline arcs (Fig. 4). The locations of the single-object display target features were generated randomly for each stimulus and each participant, constrained such that no two features could overlap. The arc orientation in the multi-object displays was randomly selected from a set of angles $\left(-20^{\circ},-10^{\circ}, 0^{\circ},+10^{\circ},+20^{\circ}\right)$ to avoid the illusory percept of a circle. Visual stimuli subtended a maximum of $9^{\circ}$ visual angle, and were presented in the same manner as Experiment 3.

Procedure The same procedure as in Experiment 1 was used, with the exception of a change in instruction and experiment length. Subjects were asked to report how many protrusions or shapes were in each display, and each block consisted of 225 trials (25 variants $\times 9$ numerosities).

Analyses The same analyses were performed as in Experiments 1 and 2. The average percent of trials removed in trimming per numerosity was $7.9 \%$.

Results We observed the subitizing elbow, represented by significantly higher counting slopes than subitizing slopes, in both the multi-object and the single-object displays (see Table 7 for Experiment 6 statistics). The 
Table 6 Statistical Results from Experiment 5

\begin{tabular}{|c|c|c|c|c|c|c|c|c|}
\hline Experiment 5 & df & T-value & p-value & Mean difference & SD & CI & Cohen's D & d-unb \\
\hline \multicolumn{9}{|l|}{ Presence of Subitizing: } \\
\hline Subitizing Elbow: Difference in slope (Counting-Subitizing) & & & & & & $90 \% \mathrm{CI}$ & & \\
\hline Multi-Object & 11 & 3.54 & .002 & $14.60 \%$ err. & 16.19 & [7.18-22.02] & 1.45 & 1.35 \\
\hline Single-Object & 11 & 5.48 & $<.001$ & $17.72 \%$ err. & 11.70 & {$[11.91-23.53]$} & 2.1 & 1.96 \\
\hline Best Fit Model: Bilinear-Exponential R2-adjusted & & & & & & $95 \% \mathrm{CI}$ & & \\
\hline Multi-Object & 11 & 4.01 & .002 & 0.09 & 0.25 & {$[0.04-0.13]$} & 0.38 & 0.36 \\
\hline Single-Object & 11 & 6.71 & $<.001$ & 0.05 & 0.14 & {$[0.04-0.07]$} & 0.64 & .59 \\
\hline \multicolumn{9}{|l|}{ Effects of Display Type: } \\
\hline Difference in subitizing range (Multi-object-single-object) & & & & & & $95 \% \mathrm{CI}$ & & \\
\hline Subitizing Range & 11 & 0.75 & .469 & 0.28 items & 1.09 & {$[-0.55-1.12]$} & 0.22 & 0.20 \\
\hline Difference in slope (Multi-object-single-object) & & & & & & $95 \% \mathrm{CI}$ & & \\
\hline Subitizing & 11 & -0.23 & $>.250$ & $0.15 \%$ err. & 2.07 & {$[-1.54-1.25]$} & -0.07 & -0.07 \\
\hline Counting & 11 & -0.96 & $>.250$ & $-3.27 \%$ err. & 13.70 & {$[-10.75-4.21]$} & -0.25 & -0.23 \\
\hline
\end{tabular}

Note: Statistical results testing the presence of subitizing and effects of display type. For the one-tailed t-tests examining the presence of a subitizing elbow, we report a two-tailed $90 \%$ CI. For all other tests we report a $95 \%$ CI. See analyses of Experiment 1 for more details. $d$-unb d-unbiased, as calculated from Cohen's-d

mean difference in slopes for both conditions had large effect sizes greater than 3 . We also tested that a bilinear model, demonstrating the presence of subitizing in the lower numerosities and lack of subitizing in higher

\section{Experiment 5}

\section{a Stimuli}

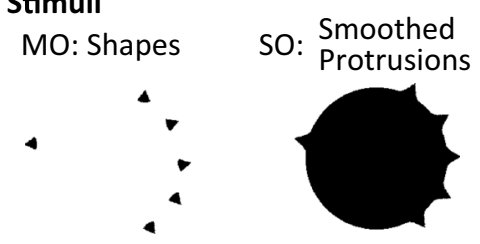

b Average Error Rate

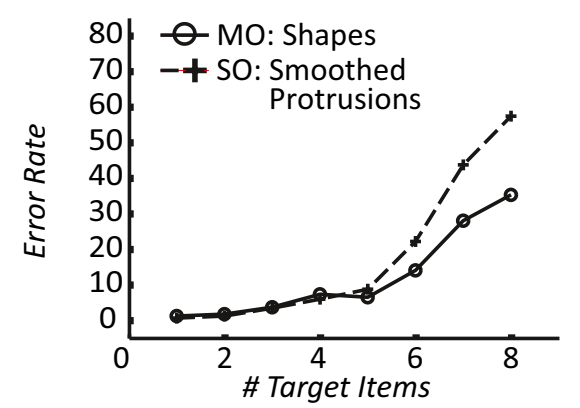

Fig. 3 Stimuli, average error rates for Experiment 5. The results for Experiment 5 are reflected here with (a) example stimuli and (b) average error rates. Average error rates are reflected in percent. $M O$ multi-object display, $S O$ single-object display numerosities, would best fit the data. While both the bilinear (multiple object: mean adjusted $\mathrm{R}^{2}=.99$, single object: mean adjusted $\mathrm{R}^{2}=.98$ ) and exponential (multiple object: mean adjusted $\mathrm{R}^{2}=.95$, single object: mean adjusted $\mathrm{R}^{2}=.96$ ) models fit the data well, a statistical comparison showed that the bilinear model better accounted for the data observed with a large effect size for both display types.

We then investigated the effects of display type on performance. The average best-fit subitizing range for the single-object displays was 3.48 smoothed protrusions, and 3.79 shapes for the multi-object displays. There was no significant difference and a small effect size for the mean difference in subitizing range across the two display types. The difference in slopes across display types was significant in the subitizing range only. See Table 2 for slopes for each condition, and Fig. 4 for reaction time and error rates.

Discussion We created these outline stimuli to avoid any percept of our single-object condition as multiple objects occluding a central circle. While there was a significant difference in subitizing slope across display types, the presence of a bilinear elbow supports subitizing in both cases. These data together with Experiments 3 and 4 suggest the participants did not view the protrusions as physically separate objects, but instead can individuate connected object parts. 
a Stimuli

\section{Experiment 6}

MO: Shapes

SO: Protrusions

D $\triangle$

$\triangle$

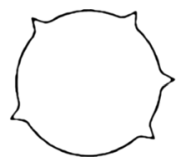

b Average Reaction Time

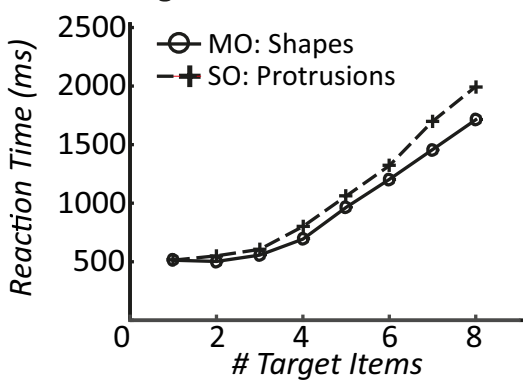

C Average Error Rate

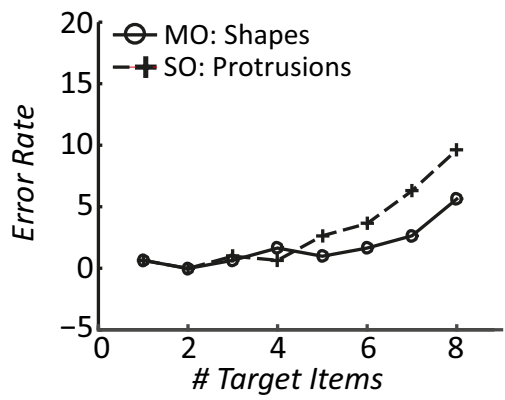

Fig. 4 Stimuli, average reaction times and error rates for Experiment 6. Results for Experiment 6 showing (a) example stimuli, (b) average reaction times, and (c) average error rates. Average reaction time data is reflected in milliseconds, and average error rates are reflected in percent. $M O$ multi-object display, $S O$ single-object display

\section{Secondary analyses}

We performed two sets of post-hoc secondary analyses, to further investigate our data.

\section{Secondary analysis 1: Secondary measures}

We performed a $2 \times 7$ repeated measures ANOVA (stimulus type $\mathrm{x}$ number) to investigate any effects present in the secondary measures of each experiment (Table 8). For experiments measuring reaction time as the measure of interest (Experiments 1-4 and 6), error rates were analyzed as the secondary measure, and vice versa for Experiment 5 which measured error rate as the primary measure. We consider these measures to be secondary, since the experimental paradigm of each experiment focused on variability of one measure. In experiments focused on reaction time, subjects were instructed to respond as quickly and accurately as possible, resulting in error rates at or close to ceiling. In Experiment 5, stimulus presentation time was limited to force errors, and subjects were allowed to respond at their own pace. This limits the amount of information available in the reaction time data.

\section{Discussion}

Overall, the results for Experiments 1-4 and 6 indicate a decrease in accuracy for the larger numerosities, although the accuracy was quite high for all numerosities and experiments. This is in line with previous studies showing that enumeration in the subitizing range is characterized by highly fast and accurate performance, whereas counting tends to be slower and more difficult (Trick \& Pylyshyn, 1994). Experiment 4 also showed a moderate interaction between stimulus type and number, although there was no significant effect of stimulus type. Experiment 5 only showed a main effect of stimulus type. This suggests that subjects tended to respond at different paces for the two stimulus types, although this is hard to interpret since the subjects were under no time explicit pressure when entering their responses.

\section{Secondary Analysis 2: Effects of slope and stimulus type}

We performed a $2 \times 2$ repeated-measures ANOVA to investigate potential interactions between stimulus type and the slope within each numerosity range (subitizing, counting) using $2 \times$ 2 ANOVAs (Table 9).

\section{Discussion}

As expected, we observed significant main effects of number range in all experiments, reflecting different slopes in the subitizing and counting ranges. In Experiments 4 and 6 there was a significant effect of stimulus type, indicating different slopes across stimulus types. The ANOVA in Experiments 1 and 4 also indicated a significant interaction, suggesting that the stimulus types can have differential effects on the slope of the subitizing and counting ranges. However, stimuli were different in both the multiple objects and single object conditions; a more pointed investigation of the effects of protrusion or stimulus type on individuation is needed to interpret these results.

\section{General discussion}

Studies of individuation have typically focused on object individuation. While most theories of object individuation agree 
Table 7 Statistical results from Experiment 6

\begin{tabular}{|c|c|c|c|c|c|c|c|c|}
\hline Experiment 6 & df & T-value & p-value & Mean difference & SD & $\mathrm{CI}$ & Cohen's D & d-unb \\
\hline \multicolumn{9}{|l|}{ Presence of Subitizing: } \\
\hline Subitizing Elbow: Difference in slope (Counting-Subitizing) & & & & & & $90 \% \mathrm{CI}$ & & \\
\hline Multi-Object & 11 & 7.10 & $<.001$ & $232.71 \mathrm{~ms}$ & 121.56 & {$[173.84-291.57]$} & 3.37 & 3.14 \\
\hline Single-Object & 11 & 11.27 & $<.001$ & $213.22 \mathrm{~ms}$ & 82.95 & [179.24-247.19] & 3.95 & 3.68 \\
\hline Best Fit Model: Bilinear-Exponential R2-adjusted & & & & & & $95 \% \mathrm{CI}$ & & \\
\hline Multi-Object & 11 & 6.45 & $<.001$ & 0.03 & 0.02 & {$[0.02-0.04]$} & 1.92 & 1.78 \\
\hline Single-Object & 11 & 5.14 & $<.001$ & 0.02 & 0.02 & {$[0.01-0.03]$} & 1.66 & 1.54 \\
\hline \multicolumn{9}{|l|}{ Effects of Display Type: } \\
\hline Difference in subitizing range (Multi-object-single-object) & & & & & & $95 \% \mathrm{CI}$ & & \\
\hline Subitizing Range & 11 & -0.97 & $>.250$ & -0.31 items & 1.10 & {$[-1.01-0.39]$} & -0.39 & -0.36 \\
\hline Difference in slope (Multi-object-single-object) & & & & & & $95 \% \mathrm{CI}$ & & \\
\hline Subitizing & 11 & -2.28 & .044 & $-41.75 \mathrm{~ms}$ & 59.49 & {$[-82.12--1.39]$} & -0.93 & -0.87 \\
\hline Counting & 11 & -1.01 & $>.250$ & $-22.26 \mathrm{~ms}$ & 70.83 & {$[-71.01-26.49]$} & -0.30 & -0.28 \\
\hline
\end{tabular}

Note: Statistical results testing the presence of subitizing and effects of display type. For the one-tailed t-tests examining the presence of a subitizing elbow, we report a two-tailed $90 \%$ CI. For all other tests we report a $95 \%$ CI. See analyses of Experiment 1 for more details. $d$-unb d-unbiased, as calculated from Cohen's-d

that items must occupy separable locations in order to be indexed (Leslie et al., 1998), it is unclear whether objecthood and parallel individuation are exclusively linked, or whether individuation can function over other categories of stimulus properties. In Experiment 1 we showed that subitization occurs over object parts that are connected to a central circle. In Experiment 2 we added irregularity to the protrusions to ensure participants did not use a single feature such as spikiness to facilitate selection. In Experiment 3 we explored whether subjects used a strategy that allowed them to visualize the protrusions as objects disconnected from the central circle. To do this, we decreased the separability of the features and added slight indents or outdents to the circle. To further ensure that subjects did not perceive the stimuli as partially occluding shapes, in Experiment 4 we removed the t-junction occlusion cue, and still observed subitization. We conclude that even when targets are perceived as parts of a whole object, subjects can individuate and enumerate the targets using the same strategy as with distinct objects. The subitization of indents in Experiment 3 is particularly interesting, as most subjects saw them as contour discontinuities and not occluding shapes. This indicates that object features do not have to appear detachable to observe subitizing behavior and for the individuation mechanism to function. We also investigated the performance of subjects when confronted with a limited presentation time in Experiment 5, and observed error rates consistent with subitization and parallel individuation for both connected object parts and physically separate objects.

One potential argument with our conclusion that parallel individuation occurs over connected object parts is that it is difficult to state unequivocally that the subjects perceived the protrusions and holes as parts of a single entity. While the majority of subjects reported seeing the target holes in Experiment 3 single-object condition as features of a single object, some perceived the targets as objects occluding the central circle. To remove the possibility that the results discussed here were a result of subjects perceiving the stimuli as many small shapes occluding a central distractor circle, we ran an experiment using line stimuli (Fig. 4). Since the targets in the single-object condition were merely deformations of a single contour, it would be very difficult for the subjects to perceive the targets as occluding a central shape. The results of Experiment 6 show evidence of subitizing and parallel individuation for the singleobject and multi-object outline stimuli, and support our conclusion that parallel individuation can occur over connected object parts.

Importantly our results suggest that the connectivity in our single-object stimuli does not eliminate the subitizing effect. This is in contrast to previous studies where connectivity interfered with estimation (Franconeri, Bemis, \& Alvarez, 2009; He et al., 2009) or multiple object tracking performance (Scholl et al., 2001). However, subitizing differs from estimation in both quantitative range and accuracy, and neural data suggests that separate mechanisms underlie the two processes (Cutini, Scatturin, Basso Moro, \& Zorzi, 2014), suggesting that connectivity may have a different impact on exact enumeration and estimation. Our results may differ from those from the MOT literature (Scholl et al., 2001) due to different task requirements. Connectivity may compete with or influence processing demands that occur later than individuation and may not be crucial for a simple enumeration task, such as the maintenance of representations over time or through space or ignoring of distractors. 
Table 8 Secondary measures

\begin{tabular}{|c|c|c|c|}
\hline Secondary Analysis 1 & df & $\mathrm{F}$ & p-value \\
\hline \multicolumn{4}{|c|}{ Experiment 1: Circles|Spikes (Error Rate) } \\
\hline Stimulus Type & $(1,13)$ & 2.94 & .11 \\
\hline Numerosity & $(1.68,21.79)$ & 5.29 & .017 \\
\hline Stimulus Type $\times$ Number & $(1.88,24.40)$ & 1.77 & .193 \\
\hline \multicolumn{4}{|c|}{ Experiment 2: Shapes|Protrusions (Error Rate) } \\
\hline Stimulus Type & $(1,12)$ & .526 & $>.250$ \\
\hline Numerosity & $(2.71,32.53)$ & 3.64 & .026 \\
\hline Stimulus Type $\times$ Number & $(3.03,36.32)$ & .598 & $>.250$ \\
\hline \multicolumn{4}{|c|}{ Experiment 3: Outdents|Indents (Error Rate) } \\
\hline Stimulus Type & $(1,11)$ & .306 & $>.250$ \\
\hline Numerosity & $(2.60,28.55)$ & 3.73 & .027 \\
\hline Stimulus Type $\times$ Number & $(2.80,30.77)$ & 1.13 & $>.250$ \\
\hline \multicolumn{4}{|c|}{ Experiment 4: Shapes|Protrusions (Error Rate) } \\
\hline Stimulus Type & $(1,16)$ & .34 & $>.250$ \\
\hline Numerosity & $(2.94,47.0)$ & 3.53 & .022 \\
\hline Stimulus Type $\times$ Number & $(7,112)$ & 2.72 & .012 \\
\hline \multicolumn{4}{|c|}{ Experiment 5: Shapes|Protrusions (Reaction Time) } \\
\hline Stimulus Type & $(1,11)$ & 122.70 & $<.001$ \\
\hline Numerosity & $(1.37,15.12)$ & 1.64 & .225 \\
\hline Stimulus Type $\times$ Number & $(7,77)$ & 1.91 & .080 \\
\hline \multicolumn{4}{|c|}{ Experiment 6: Outline Shapes|Protrusions (Error Rate) } \\
\hline Stimulus Type & $(1,11)$ & 2.31 & .157 \\
\hline Numerosity & $(1.66,18.20)$ & 5.87 & .014 \\
\hline Stimulus Type $\times$ Number & $(2.11,23.22)$ & 1.52 & .239 \\
\hline
\end{tabular}

Note: Statistical results of a $2 \times 7$ repeated measures ANOVA performed over the secondary measures of each experiment. A Greenhouse-Geisser correction was applied when conditions of sphericity were not met

While connectivity may not interfere with individuation in the case of our single-object stimuli, connectivity plays an important role in the parsing and selection of items in the visual field. Connectivity is a strong cue when segmenting the visual field into units, and has been proposed as the cue resulting in the initial organization of visual field, after which other grouping or parsing can occur (Palmer \& Rock, 1994). This theory has since garnered support, with segmentation of units based on uniform connectedness (Palmer \& Rock, 1994) occurring faster than grouping by similarity, and as fast as grouping by proximity (Han, Humphreys, \& Chen, 1999). Connectivity also has shown to be a very important cue with regards to perception and how attention is allocated, as evidenced by Balint's syndrome. Merely adding a connecting line between two shapes allows patients to perform a task comparing features of the shapes, which was otherwise impossible (Humphreys \& Riddoch, 1993, p. 158). For these reasons we believe that our finding that parallel individuation does occur over connected object parts is not trivial, and is somewhat unexpected given the interference present in other tasks (Franconeri, Bemis, \& Alvarez, 2009; He et al., 2009; Scholl et al., 2001).
Table 9 Secondary measures

\begin{tabular}{|c|c|c|c|}
\hline Secondary Analysis 2 & df & $\mathrm{F}$ & p-value \\
\hline \multicolumn{4}{|c|}{ Experiment 1: Circles|Spikes (Reaction Time) } \\
\hline Stimulus Type & $(1,13)$ & .376 & $>.250$ \\
\hline Number Range & $(1,13)$ & 55.39 & $<.001$ \\
\hline Stimulus Type $\times$ Range & $(1,13)$ & 5.10 & .042 \\
\hline \multicolumn{4}{|c|}{ Experiment 2: Shapes|Protrusions (Reaction Time) } \\
\hline Stimulus Type & $(1,12)$ & .686 & $>.250$ \\
\hline Number Range & $(1,12)$ & 94.75 & $<.001$ \\
\hline Stimulus Type $\times$ Range & $(1,12)$ & 1.91 & .192 \\
\hline \multicolumn{4}{|c|}{ Experiment 3: Outdents|Indents (Reaction Time) } \\
\hline Stimulus Type & $(1,11)$ & 1.99 & .186 \\
\hline Number Range & $(1,11)$ & 69.48 & $<.001$ \\
\hline Stimulus Type $\times$ Range & $(1,11)$ & .130 & $>.250$ \\
\hline \multicolumn{4}{|c|}{ Experiment 4: Shapes|Protrusions (Reaction Time) } \\
\hline Stimulus Type & $(1,16)$ & 4.49 & .050 \\
\hline Number Range & $(1,16)$ & 147.39 & $<.001$ \\
\hline Stimulus Type $\times$ Range & $(1,16)$ & 11.46 & .004 \\
\hline \multicolumn{4}{|c|}{ Experiment 5: Shapes|Protrusions (Error Rate) } \\
\hline Stimulus Type & $(1,11)$ & .965 & $>.250$ \\
\hline Number Range & $(1,11)$ & 24.15 & $<.001$ \\
\hline Stimulus Type $\times$ Range & $(1,11)$ & .824 & $>.250$ \\
\hline \multicolumn{4}{|c|}{ Experiment 6: Outline Shapes|Protrusions (Reaction Time) } \\
\hline Stimulus Type & $(1,11)$ & 7.09 & .022 \\
\hline Number Range & $(1,11)$ & 111.17 & $<.001$ \\
\hline Stimulus Type $\times$ Range & $(1,11)$ & .353 & $>.250$ \\
\hline
\end{tabular}

Note: Results of a $2 \times 2$ Repeated measures ANOVA, with stimulus type and slope of the two ranges (subitizing, counting) as factors. A Greenhouse-Geisser correction was applied when conditions of sphericity were not met

While we successfully showed that objecthood, as defined by lack of connectivity, is not a necessary requirement for individuation, we still do not have a precise definition of the limitations on the segmentation process. The target items in our stimuli all occupied non-overlapping locations in space, however the exact influence of crowding or overlap on the subitization process is still relatively unknown. Atkinson, Campbell, and Francis (1976) found that manipulating the spatial frequency of objects affected the subitizing limit. In a task requiring subjects to track the featural changes of two gabors overlaid in the same space, subjects were not able to successfully attend to and track the features of both gabors (Blaser, Pylyshyn, \& Holcombe 2000). It thus seems as though overlapping stimuli cannot be individuated and tracked in parallel, consistent with the hypothesis that occupying a distinct location in space is critical for individuation to occur. Parallel individuation may require a minimum distance between targets, but not require the targets to be disconnected in the context of rapid enumeration. Furthermore, some feature elements other than connectivity may facilitate or inhibit 
the individuation process more than others, which could explain the difference in subitizing slope across display types we observed in Experiments 4 and 6. Further investigations directed at investigating the modulation of the subitizing effect will help clarify the effect of other features on the individuation process.

While we have emphasized the importance of targets occupying separate locations, one study suggests that intermixed and spatially overlapping groups of targets can be enumerated in parallel (Halberda, Sires, \& Feigenson, 2006). This study presented displays consisting of colored dots to participants, and asked them to enumerate subsets of the dots based on their color. Participants successfully enumerated up to three sets of dots, and the authors suggested that groups of dots, as defined by color, could be selected as individuals, with numerosity stored as a feature of those individuals. The overlapping quality of stimuli may or may not interfere with numerosity processing, depending on whether the task requires exact enumeration or estimation. The numerosites stored as features for the stimulus sets in Halberda et al. (2006) fell outside of the subitizing range, making it most probable that the numerosity features stored for each set were obtained using estimation. Overlapping stimuli may affect performance in parallel individuation but not in estimation.

In order to reconcile how parallel individuation could occur over parts of a single connected object, we must consider how the selection of targets could occur. All targets could also be selected as deviations from a frame of reference on some dimension. In the multi-object condition, each object differed in luminance from the background. In the single-object condition, all targets were deviations from the circular form. The more specific frame of reference in the single-object condition may have changed the critical dimension from "deviation from background" to "deviation from circle," or "deviation in color" to "deviation in curvature."

"Deviating from X frame of reference" may be described in terms of discriminability - both spatial and perceptual. The results reported here support an individuation mechanism functioning over items that are both unique in location and perceptually discriminable from a flexible frame of reference. The constraints on this discriminability are still undefined. We know that when distracters are present, the target items must deviate on a single dimension rather than be selected through a conjunction - such as color AND orientation (Trick \& Pylyshyn, 1993). What constitutes a single dimension however, remains ambiguous; are indents and outdents on opposing ends of the same scale, or separate dimensions?

The idea of a flexible frame of reference directly relates to the issue of "objecthood" at various hierarchical stages of a scene. Feldman (2003) suggests that strong cues for objecthood result from non-accidental relationships existing at a level one step below a more global parsing of the scene where only accidental relationships exist between parsed units. According to Feldman's theory, the strength of objecthood depends upon the disparity between the number of non-accidental relationships at adjacent levels of the scene hierarchy (2003). The single-object stimuli used in our study has one more level in its hierarchical structure than the multi-object condition. However, the difference in non-accidental properties between the object parts and the whole object still seems to be significant enough to allow for parallel individuation to occur.

Within the broader context of the subitizing literature, parallel individuation has already been shown to occur over several levels of a scene hierarchy. Trick and Enns (1997) demonstrated that both grouped dots located at the vertices of invisible shapes and line-drawn shapes can be subitized. Our experiment expanded their results to show that not only can subitization occur over groups and distinct objects, but it can also occur over parts of a single object.

Simply because parallel individuation can occur over multiple levels of a hierarchical tree does not require each level to be processed equally well in all contexts. When distracting elements were added to the grouped stimuli, subitizing was no longer present (Trick \& Enns, 1997). Our results do not speak to how individuation occurs over connected object parts in the context of distractors. We also limited our investigation to identifying the presence of subitizing in different stimulus types, and did not directly manipulate or test the effectiveness of individuating different stimulus types or protrusions. We investigated potential interactions between stimulus type and numerosity range in our data post-hoc using $2 \times 2$ ANOVAs (Secondary Analysis 2) and observed a significant main effect of stimulus type in Experiments 4 and 6, and significant interactions in Experiments 1 and 4, suggesting that various stimulus features can have a differential effect on the slope, either for both ranges, or more so on one numerosity range. The effect of different features on the subitizing slope is an important question that begs further study. In the present study, we observed a range of slopes within the experiments presented here. However, we feel confident that the variation in slope does not reflect a lack of parallel individuation. In fact, a variance in slope is typically reported in the subitizing literature. In subitizing " $\ldots$.each additional item may add 40-100 ms. When there are more than 4 items, the slope jumps to $250-350 \mathrm{~ms} /$ item." (Trick \& Pylyshyn, 1994, p. 80). All of our results fall nicely within those ranges.

An investigation into how different object parts may affect subitizing performance would relate to the pattern-matching theory of subitizing (Mandler \& Shebo, 1982), where targets form vertices of shapes that can then be matched to a number concept. A recent study showing that subitizing performance cannot be explained by the probability of detecting target items supports this theory (Palomares \& Egeth, 2010). The importance of the overall shape has also been emphasized in a study of the effects of the medial axis or skeletal structure of a shape on the perception of texture (Harrison \& Feldman, 2009). Harrison and Feldman's results suggest that when the skeleton of a shape is consistent with internal local features, 
perception of those local features is enhanced. For the singleobject stimuli used in the present study, the type of uniform connectivity added by the central circle would not dramatically affect the skeleton of the shape, since the protruding targets define the skeleton shape. Thus a theory of subitizing that relies on pattern-matching would suggest equal performance for both multi-object and single-object stimuli. However, altering the skeleton or gestalt of the single-object stimuli may affect the individuation of the target parts differently than the multiobject stimuli. For example, if the protruding stimuli were manipulated to be misaligned with the internal structure, then we could observe worse performance than similarly rotated targets in a multi-object display. This is an open question.

Further investigations such as the ones described above will help winnow out different theories of parallel individuation. A careful manipulation of object parts could provide results that speak to the validity of pattern-matching theories. The type of object part could also affect ease of selection. Requiring a target to have certain qualities on a particular dimension affords some tension with theories that describe individuation of an item as occurring without knowledge of its features (Leslie et al., 1998; Pylyshyn, 1989). If an item is selected as "unique," how does that occur without identifying what dimension reflects the difference? It may be useful to consider our stimuli and those of future experiments within the framework of "textons." Textons are visual stimuli that can be discriminated preattentively without knowledge of the relevant positioning of the items (Julesz, 1984). It is possible that the individuation mechanism may be the joint result of information from a perceptual texton detector and spatial location discriminator. If we combine our emphasis on location with the discriminability of textons, we may have a working definition of individuation that does not rely on objecthood.

Previously, parallel individuation has been nearly synonymous with parallel object individuation. While objecthood clearly provides important information that many tasks depend upon, such as multiple-object tracking, it does not appear to be a necessary requirement in all situations. Within the context of subitizing, we showed that deviations occupying distinct locations on a single connected object provide enough information for parallel individuation to occur. These results are unexpected given the behavioral results of other tasks relying on individuation. Future investigations on how parallel individuation occurs and is influenced by different task demands should consider individuation as not necessarily bound to objecthood or connectivity, and allow for the possibility of individuation functioning over different locations merely on the basis of local discontinuity.

\section{Appendix}

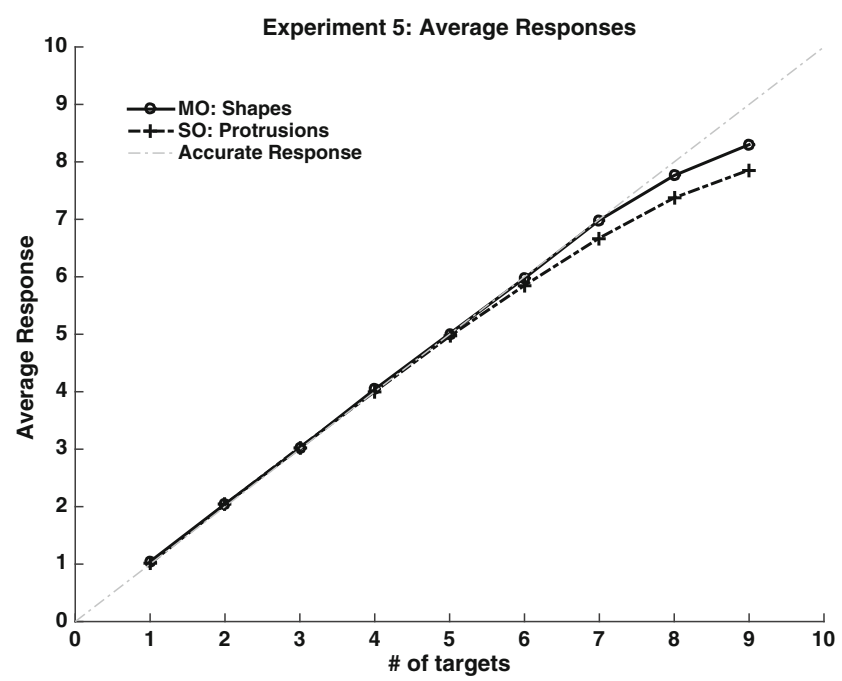

Fig. 5 Average responses for each display type in Experiment 5. The gray dotted line indicates the correct response. $M O$ multi-object display, $S O$ single-object display

\section{References}

Akin, O., \& Chase, W. (1978). Quantification of three-dimensional structures. Journal of Experimental Psychology: Human Perception and Performance, 4(3), 397-410.

Alvarez, G. A., \& Cavanagh, P. (2005). Independent resources for attentional tracking in the left and right visual hemifields. Psychological Science, 16(8), 637-643.

Atkinson, J., Campbell, F. W., \& Francis, M. R. (1976). The magic number $4 \pm 0$ : A new look at visual numerosity judgments. Perception, 5 , 327-334.

Balakrishnan, J. D., \& Ashby, F. G. (1991). Is subitizing a unique numerical ability? Perception \& Psychophysics, 50, 555-564.

Blaser, E., Pylyshyn, Z. W., \& Holcombe, A. O. (2000). Tracking an object through feature space. Nature, 408(9), 196-199.

Brainard, D. H. (1997). The psychophysics toolbox. Spatial Vision, 10, 433-436.

Brase, G. L., Cosmides, L., \& Tooby, J. (1998). Individuation, counting, and statistical inference: The role of frequency and whole-object representations in judgment under uncertainty. Journal of Experimental Psychology: General, 127(1), 3-21.

Chesney, D. L., \& Haladjian, H. H. (2011). Evidence for a shared mechanism used in multiple-object tracking and subitizing. Attention, Perception, \& Psychophysics, 73(8), 2457-2480.

Cohen, J. (1988). Statistical power analysis for the behavioral sciences (2nd ed.). Hillsdale: Erlbaum.

Cummings, G. (2012). Understanding the new statistics: Effect sizes, confidence intervals, and meta-analysis. New York: Routledge. ISBN 978-0-415-87967-5.

Cutini, S., Scatturin, P., Moro, S. B., \& Zorzi, M. (2014). Are the neural correlates of subitizing and estimation dissociable? An fNIRS investigation. NeuroImage, 85, 391-399.

Ester, E. F., Drew, T., Klee, D., Vogel, E. K., \& Awh, E. (2012). Neural measures reveal a fixed item limit in subitizing. The Journal of Neuroscience, 32(21), 7169-7177. 
Faul, F., Erdfelder, E., Lang, A., \& Buchner, A. (2007). G*Power 3: A flexible statistical power program for the social, behavioral, and biomedical sciences. Behavior Research Methods, 39, 175-191. doi:10.3758/BF03193146

Feldman, J. (2003). What is a visual object? TICS, 7(6), 252-256.

Franconeri, S. L., Bemis, D. K., \& Alvarez, G. A. (2009). Number estimation relies on a set of segmented objects. Cognition, 113, 1-13.

Giralt, N., \& Bloom, P. (2000). How special are objects? Children's reasoning about objects, parts, and holes. Psychological Science, 11(6), 497-501.

Halberda, J., Sires, S. F., \& Feigenson, L. (2006). Multiple spatially overlapping sets can be enumerated in parallel. Psychological Science, 17(7), 572-576.

Han, S., Humphreys, G. W., \& Chen, L. (1999). Uniform connectedness and classical Gestalt principles of perceptual grouping. Perception \& Psychophysics, 61(4), 661-674.

Harrison, S. J., \& Feldman, J. (2009). The influence of shape and skeletal axis structure on texture perception. Journal of Vision, 9(6), 13.

He, L., Zhang, J., Zhou, T., \& Chen, L. (2009). Connectedness affects dot numerosity judgment: Implications for configural processing. Psychonomic Bulletin \& Review, 16(3), 509-517.

Humphreys, G. W., \& Riddoch, M. J. (1993). Interactions between object and space systems revealed through neuropsychology. Attention and Performance, XIV, 143-162.

Julesz, B. (1984). A brief outline of the texton theory of human vision. TINS, 7, 41-45.

Kaufman, E. L., Lord, M. W., Reese, T. W., \& Volkmann, J. (1949). The discrimination of visual number. The American Journal of Psychology, 62(4), 498-525.

Kleiner, M., Brainard, D., \& Pelli, D. (2007). What's new in Psychtoolbox-3? Perception, 36, ECVP Abstract Supplement.

Kraft, J. M., \& Brainard, D. H. (1999). Mechanisms of color constancy under nearly natural viewing. PNAS, 96, 307-312.

Leslie, A. M., Xu, F., Tremoulet, D., \& Scholl, B. J. (1998). Indexing and the object concept: developing 'what' and 'where' systems. TICS, 2(1), 10-18.

Logan, G. D., \& Zbrodoff, N. J. (2003). Subitizing and similarity: Toward a pattern-matching theory of enumeration. Psychonomic Bulletin \& Review, 10(3), 676-682.

Mandler, G., \& Shebo, B. J. (1982). Subitizing: An analysis of its component processes. Journal of Experimental Psychology: General, 111(1), 1.

Marr, D. (1982). Vision. New York: Freedman.

Nelder, J. A., \& Mead, R. (1965). A simplex method for function minimization. The Computer Journal, 7(4), 308-313.

Pagano, S., Lombardi, L., \& Mazza, V. (2014). Brain dynamics of attention and working memory engagement in subitizing. Brain Research, 1543, 244-252.

Palmer, S., \& Rock, I. (1994). Rethinking perceptual organization: The role of uniform connectedness. Psychonomic Bulletin and Review, l(1), 29-55.
Palomares, M., \& Egeth, H. (2010). How element visibility affects visual enumeration. Vision Research, 50(19), 2000-2007.

Pelli, D. G. (1997). The VideoToolbox software for visual psychophysics: Transforming numbers into movies. Spatial Vision, 10, 437-442.

Pylyshyn, Z. (1989). The role of location indexes in spatial perception: A sketch of the FINST spatial-index model. Cognition, 32(1), 65-97.

Pylyshyn, Z. W., \& Storm, R. W. (1988). Tracking multiple independent targets: Evidence for a parallel tracking mechanism*. Spatial Vision, 3(3), 179-197.

Railo, H. (2014). Bilateral and two-item advantage in subitizing. Vision Research, 103, 41-48.

Rubin, N. (2001). The role of junctions in surface completion and contour matching. Perception, 30, 339-366.

Scholl, B. J., \& Pylyshyn, Z. W. (1999). Tracking multiple items through occlusion: Clues to visual objecthood. Cognitive Psychology, 38(2), 259-290.

Scholl, B. J., Pylyshyn, Z. W., \& Feldman, J. (2001). What is a visual object? Evidence from target merging in multiple object tracking. Cognition, 80, 159-177.

Shipley, E. F., \& Shepperson, B. (1990). Countable entities: Developmental changes. Cognition, 34(2), 109-136.

Sonderegger, D. (2012). SiZer: SiZer: Significant zero crossings. R package version 0.1-4. R website. Avaliable at: http://www.r-project.org

Spelke, E. S. (1990). Principles of object perception. Cognitive Science, 14, 29-56.

Spelke, E. S., Kestenbaum, R., Simons, D., \& Wein, D. (1995). Spatiotemporal continuity, smoothness of motion and object identity in infancy. The British Journal of Developmental Psychology, 13, $113-142$.

Trick, L. M., \& Enns, J. T. (1997). Clusters precede shapes in perceptual organization. Psychological Science, 8(2), 124-129.

Trick, L. M., \& Pylyshyn, Z. W. (1993). What enumeration studies can show us about spatial attention: Evidence for limited capacity preattentive processing. Journal of Experimental Psychology: Human Perception and Performance, 19, 331-351.

Trick, L. M., \& Pylyshyn, Z. W. (1994). Why are small and large numbers enumerated differently? A limited-capacity preattentive stage in vision. Psychological Review, 101(1), 80-102.

Van de Walle, G. A., Carey, S., \& Prevor, M. (2000). Bases for object individuation in infancy: Evidence from manual search. Journal of Cognition and Development, 1(3), 249-280.

vanMarle, K., \& Scholl, B. J. (2003). Attentive tracking of objects versus substances. Psychological Science, 14(5), 498-504.

Watson, D. G., \& Maylor, E. A. (2006). Effects of color heterogeneity on subitization. Perception \& Psychophysics, 68(2), 319-326.

Watson, D. G., Maylor, E. A., \& Bruce, L. A. M. (2005). The efficiency of feature-based subitization and counting. Journal of Experimental Psychology: Human Perception and Performance, 31(6), 1449-1462.

Xu, F., Carey, S., \& Welch, J. (1999). Infants' ability to use object kind information for object individuation. Cognition, 70(2), 137-166. 